\title{
Tophaceous Gout of the Spine Causing Neural Compression
}

\author{
Jong-Won Yoon ${ }^{1}$, Kyung-Bum Park ${ }^{1}$, Hyun Park ${ }^{1}$ Dong-Ho Kang ${ }^{1}$, Chul-Hee Lee , \\ Soo-Hyun Hwang ${ }^{1}$, Jin-Myung Jung ${ }^{1}$, Jong-Woo Han ${ }^{1}$, In Sung Park ${ }^{1,2}$ \\ ${ }^{I}$ Department of Neurosurgery, Gyeongsang National University School of Medicine, \\ ${ }^{2}$ Gyeongsang Institute of Health Science, Jinju, Korea
}

Gout is a common metabolic disease in which monosodium urate crystals called tophi develop. Spinal involvement in gout resulting in neural compression is unusual. We describe a case of a 64-year-old man with a history of gouty arthritis of the knee. The patient presented with thoracic myelopathy and radiculopathy. Imaging of the spine revealed an extradural mass lesion with bony erosion of the thoracic spine. A decompressive operation was performed, and a chalky white material was found. Histopathological examination confirmed a gouty tophus. The symptoms of spinal gout vary and its radiological features are not sufficiently specific to provide a definite diagnosis. Therefore, in patients with a history of gouty arthritis who present with neural compressive symptoms of the spine, spinal gout should be strongly suspected.

Key Words: Gout $\cdot$ Spine $\cdot$ Myelopathy $\cdot$ Radiculopathy

\section{INTRODUCTION}

Gout is a common metabolic disease in which deposition of monosodium urate (MSU) crystals causes inflammatory arthritis of the peripheral joints. Nodules formed by the accumulation of MSU crystals in the soft tissue are called tophi. Gout affects $1-2 \%$ of adults in developed countries, where it is the most common type of inflammatory arthritis in men $^{13)}$. Typically, gout affects the distal joints of the appendicular skeleton. The occurrence of gout in the spine has been reported in only a limited number of cases ${ }^{1,2,5,6,8-12,14,16,18)}$.

In this report, we describe a patient who complained of paraparesis. The cause was a cord-compressing mass-like lesion at the thoracic level. The presumed diagnosis according to the imaging was a neoplastic lesion, but histopathological examination confirmed the diagnosis as a gouty tophus.

\section{CASE REPORT}

A 64-year-old man was admitted to our clinic with week-

- Received: August 2, 2013 - Revised: September 9, 2013

- Accepted: September 11, 2013

Corresponding Author: Kyung-Bum Park, MD

Department of Neurosurgery, Gyeongsang National University Hospital,

90 Chiram-dong, Jinju, Gyeongsangnam-do 660-702, Korea

Tel: +82-55-750-8112, Fax: +82-55-759-0817

E-mail: kongs70@hanmail.net long weakness of both lower extremities. The patient also complained of back pain radiating to his left anterior chest for 4 weeks. Eight years prior, the patient had acute gout arthritis of the right knee. At that time, the symptoms spontaneously resolved, and the patient received no subsequent management for the condition. The back and chest pain had increased gradually and had become more severe over the previous month in spite of conservative treatment. A week before admission, paraparesis progressed to difficulty walking. The patient had no sphincter function impairment, fever, or weight loss.

Neurological examination showed paraparesis with grade IV motor weakness of both the hip and knee with no sensory impairment. Reflexes were normoactive and within normal ranges as assessed using the straight leg raise test. The patient's white blood cell count was slightly increased to $10,960 / \mathrm{mm}^{3}$

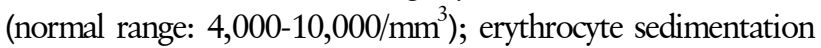
rate was $5 \mathrm{~mm} / \mathrm{h}$ (normal range: lower than $9 \mathrm{~mm} / \mathrm{h}$ ); C-reactive protein level was $0.9 \mathrm{mg} / \mathrm{dL}$ (normal range: lower than $0.5 \mathrm{mg} / \mathrm{dL}$ ); and serum uric acid level was $12.2 \mathrm{mg} / \mathrm{dL}$ (normal range: lower than $7.0 \mathrm{mg} / \mathrm{dL}$ ). No symptoms of gouty arthritis such as pain or swelling were reported for other joints of the appendicular skeleton.

Computed tomography (CT) demonstrated a low-density mass with calcification surrounding the T6/7 left facet articulation and T6 and T7 left lamina. Bony erosion was also observed at the T6/7 left facet articulation (Fig. 1). Magnetic resonance imaging (MRI) of the thoracic spine revealed an extradural mass compressing the posterior of the spinal cord at 


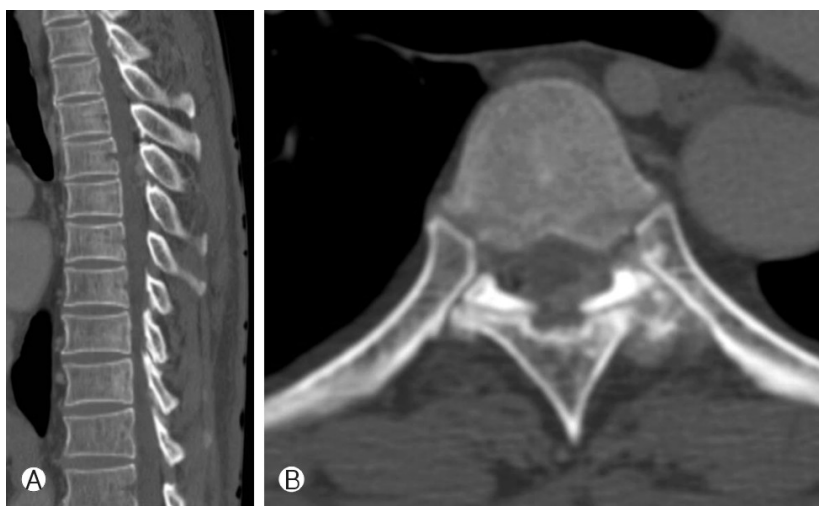

Fig. 1. Sagittal (A) and axial (B) computed tomography scans show a juxta-articular low density mass with calcification and bony erosion at the T6/7 left facet articulation.

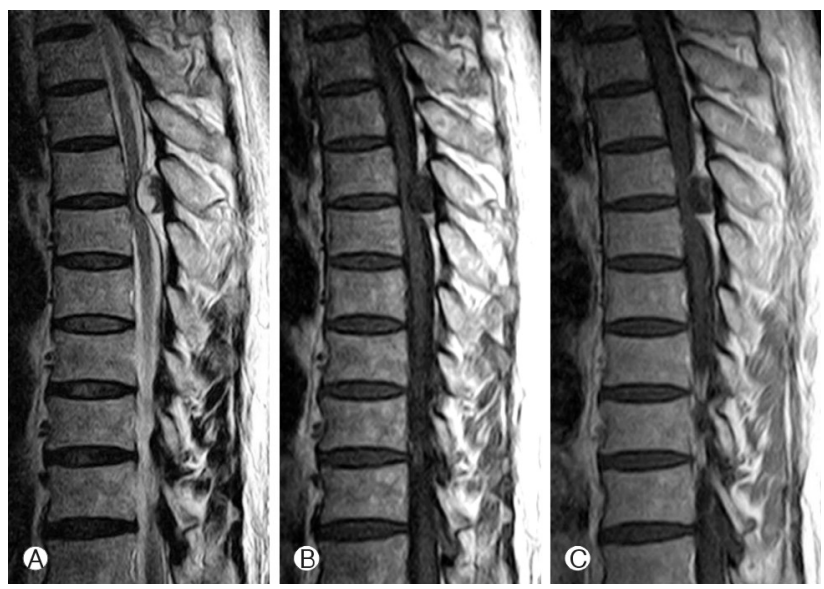

Fig. 2. Magnetic resonance imaging findings. (A) T2-weighted image. (B) $\mathrm{Tl}$-weighted image. (C) Contrast-enhanced $\mathrm{Tl}$-weighted image.

T5-7, with heterogeneous high and low signal on T2-weighted images and low signal on T1-weighted images. The small amount of the low signal intensity of the mass on T2-weighted MRI corresponded to the calcified lesion on CT, and this lesion showed peripheral enhancement on post-contrast MRI (Fig. 2).

We made a tentative diagnosis based on the imaging findings of an epidural cyst or benign bone-originating tumor. Thoracic laminectomy from T5 to T7 was performed for the purpose of neural decompression and for histopathological confirmation. Intraoperatively, a chalky white material was found at the interlaminar space. This material extended to the dorsal space of the thoracic dura and the T5/6, T6/7 left foramen (Fig. 3). After left T5/6, T6/7 facetectomy, the mass that was compressing the cord and root was removed and T5, 6, and 7 both pedicle screw fixation with posterolateral fusion was performed.

Histopathological examination of the specimen by polar-
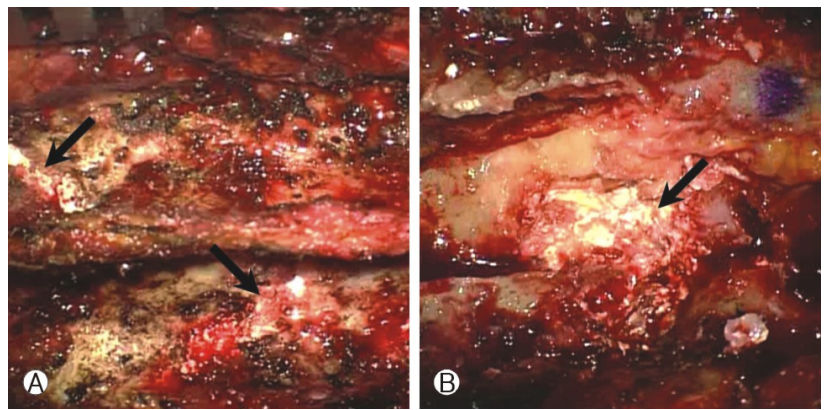

Fig. 3. Intraoperative photograph of pre-laminectomy (A) and postlaminectomy and facetectomy (B). Chalky whitish tophus (black arrows) was seen at the T5/6 and 6/7 interlaminar space (A) and epidural space under the ligamentum flavum extending to the T5/6 neural foramen (B).
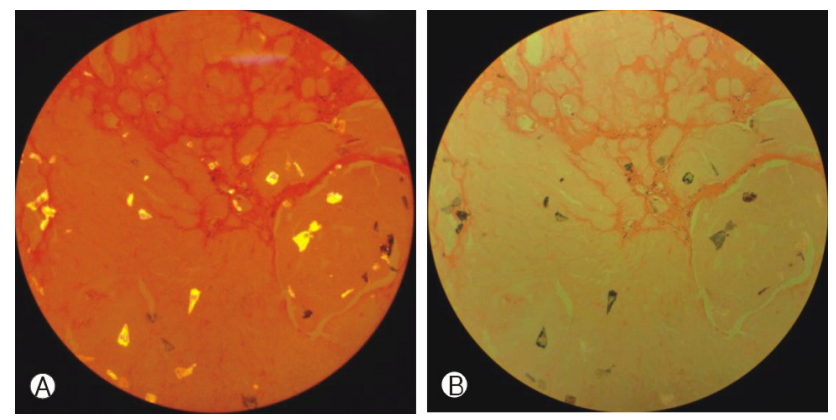

Fig. 4. Polarized light microscopy findings of the specimen. The crystal demonstrates strong negative birefringence: yellow when aligned parallel to the axis $(A)$ and blue when aligned across the direction of polarization (B).

ized light microscope demonstrated a granular material with a needle pattern and negative birefringence (Fig. 4). This finding confirmed a diagnosis of a gouty tophus. No neoplastic tissue was found in the specimen. Postoperatively, the patient regained strength in both lower extremities, and his back and chest pain attenuated. After being discharged from the hospital, the patient has been treated with medication.

\section{DISCUSSION}

Gout, a purine metabolism disorder, is a common cause of inflammatory arthritis. Gout is caused by hyperuricemia leading to production of MSU crystals. Gout typically affects the peripheral joints of the appendicular skeleton but is also known to involve the axial joints. Although involvement of the spine is an unusual manifestation of tophaceous gout, spinal gout involvement is probably under-diagnosed because asymptomatic patients with this condition are unlikely to undergo neuroimaging of the spine ${ }^{8)}$. A recent study found evi- 
dence of axial involvement, with or without symptoms, in 9 of 64 patients with gouty arthritis who had undergone a spinal CT examination ${ }^{10)}$. However, only a few cases of histologically proven gout causing spinal cord compression have been reported in the literature.

Spinal involvement of gouty tophus has been observed in all spinal sections. The most frequent involvement reported in the literature is the lumbar spine ${ }^{10,14)}$. However, the cervical and thoracic spine can also be affected ${ }^{1,4,5,15,17)}$. Tophaceous crystal deposition in axial elements of the spine is generally found at the facet articulation, but the ligamentum flavum, pedicle, lamina, epidural soft tissue, vertebral body, and disc space can also be affected ${ }^{11,12,16}$. Chang et al. suggested that tophaceous accumulation begins at the facet joint and spreads to the ligamentum flavum and other elements"

Patients with symptomatic gouty spine can present with spinal pain and fever associated with local and systemic inflammatory response. Because of the inflammation and the radiological findings, spinal gout is often mischaracterized as spondylitis or epidural abscess, as described in the literature ${ }^{16,18)}$. Furthermore, the formation of a tophus can cause varied neurological manifestations that depend on the nature of the neural compression such as radiculopathy, claudication, and myelopathy $^{4,5,8-10,12,15-17)}$.

The radiological features of spinal gout are nonspecific and varied. Standard radiographs can be normal or might reveal degenerative changes such as disc-space narrowing and bony erosion. MSU crystals are not radiographically opaque so unless the urate deposits calcify, standard radiographs are not sensitive enough to detect them and therefore have limited use in diagnosing spinal gout ${ }^{6,10)}$. CT and MRI are more specific imaging methods, but lack the accuracy required for differential diagnosis of spinal gout. CT can show juxta-articular masses with low-density and intra-articular and vertebral bony erosion with sclerotic or normal bony density ${ }^{16,18}$. When these features are accompanied by calcification, crystals can be seen as regions of hyperdensity on CT images ${ }^{6}$. On MRI, a tophus can have various appearances, with intermediate-to-low signal intensity on T1-weighted images and variable signal intensity on T2-weighted images. Contrast-enhanced T1-weighted MRI has two enhancement patterns: homogeneous enhancement and heterogeneous peripheral enhancement ${ }^{2,718)}$. In our case, CT revealed a low-density tophus, including calcification surrounding facet articulation and lamina with bony erosion; MRI showed a mass with homogeneous low signal intensity on T1-weighted images and heterogeneous low and high signal intensity on T2-weighted images. The calcified portion of the tophus on the CT images corresponded to the location of the lesion that had low signal intensity on the T2-weighted image and peripheral enhancement in a gadolinium-enhanced
T1-weighted image. These findings are delineating characteristics for tophaceous spinal gout, but spinal gout was not initially suspected. In the literature, many cases of spinal gout are misdiagnosed as other diseases such as spondylitis, epidural abscess, neoplastic lesion, or degenerative stenosis ${ }^{2,4,8,10,12,16-18)}$. Spinal gout has diverse clinical presentations, and radiological findings are not sufficiently specific to confirm a diagnosis; therefore, clinicians should consider spinal gout as a potential diagnosis for patients with a history of gout who present with spinal pain and neural compressive symptoms.

A definitive diagnosis of spinal gout requires histopathological examination. MSU crystals are easily detected under a light microscope, but the use of a polarized light microscope is required for definitive confirmation. Under polarized light, the crystals exhibit strong negative birefringence: they are yellow when aligned parallel to the axis and blue when aligned across the direction of polarization. A finding of negatively birefringent urate crystals firmly establishes the diagnosis of gouty arthritis ${ }^{13,14)}$.

Standard management of gout is stabilization of acute attacks by colchicines, non-steroidal anti-inflammatory drugs, or both, with urate-lowering therapy using allopurinol ${ }^{13)}$. In spinal gout causing neural-compressive manifestations such as myelopathy or radiculopathy, however, surgical decompression in conjunction with pharmacological treatment can provide a more satisfactory outcome $e^{3,14)}$.

\section{CONCLUSION}

Spinal gout is not easily detected because its symptoms are varied and its radiological characteristics are not specific enough to establish a definitive diagnosis. Therefore, when patients with prior episodes of gouty arthritis have neural compressive symptoms of the spine, spinal gout should be considered. For patients with neurological symptoms, neurosurgical decompression with pharmacological treatment should be performed and can provide positive outcomes.

\section{REFERENCES}

1. Beier CP, Hartmann A, Woertgen C, Brawanski A, Rothoerl $\mathrm{RD}$ : A large, erosive intraspinal and paravertebral gout tophus. Case report. J Neurosurg Spine 3:485-487, 2005

2. Bonaldi VM, Duong H, Starr MR, Sarazin L, Richardson J: Tophaceous gout of the lumbar spine mimicking an epidural abscess: Mr features. AJNR Am J Neuroradiol 17:1949-1952, 1996

3. Chang IC: Surgical versus pharmacologic treatment of intraspinal gout. Clin Orthop Relat Res:106-110, 2005

4. Diaz A, Porhiel V, Sabatier P, Taha S, Ragragui O, Comoy J, 
et al: tophaceous gout of the cervical spine, causing cord compression. Case report and review of the literature. Neurochirurgie 49:600-604, 2003

5. Draganescu M, Leventhal LJ: Spinal gout: Case report and review of the literature. J Clin Rheumatol 10:74-79, 2004

6. Hou LC, Hsu AR, Veeravagu A, Boakye M: Spinal gout in a renal transplant patient: A case report and literature review. Surg Neurol 67:65-73; discussion 2007

7. Hsu CY, Shih TT, Huang KM, Chen PQ, Sheu JJ, Li YW: Tophaceous gout of the spine: Mr imaging features. Clin Radiol 57: 919-925, 2002

8. Kelly J, Lim C, Kamel M, Keohane C, OSullivan M: Topacheous gout as a rare cause of spinal stenosis in the lumbar region: Case report. J Neurosurgery Spine 2:215-217, 2005

9. King JC, Nicholas C: Gouty arthropathy of the lumbar spine: A case report and review of the literature. Spine (Phila Pa 1976) 22:2309-2312, 1997

10. Konatalapalli RM, Demarco PJ, Jelinek JS, Murphey M, Gibson $\mathrm{M}$, Jennings $\mathrm{B}$, et al: Gout in the axial skeleton. J Rheumatol 36:609-613, 2009

11. Lam HY, Cheung KY, Law SW, Fung KY: Crystal arthropathy of the lumbar spine: A report of 4 cases. J Orthop Surg (Hong Kong) 15:94-101, 2007

12. Nakajima A, Kato Y, Yamanaka H, Ito T, Kamatani N: Spinal tophaceous gout mimicking a spinal tumor. J Rheumatol 31: 1459-1460, 2004

13. Richette P, Bardin T: Gout. Lancet 375:318-328, 2010

14. Saketkoo LA, Robertson HJ, Dyer HR, Virk ZU, Ferreyro HR, Espinoza LR: Axial gouty arthropathy. Am J Med Sci 338:140146, 2009

15. Souza AW, Fontenele S, Carrete H, Jr., Fernandes AR, Ferrari AJ: Involvement of the thoracic spine in tophaceous gout. A case report. Clin Exp Rheumatol 20:228-230, 2002

16. Suk KS, Kim KT, Lee SH, Park SW, Park YK: Tophaceous gout of the lumbar spine mimicking pyogenic discitis. J Spine 7:9499, 2007

17. Yen HL, Cheng CH, Lin JW: Cervical myelopathy due to gouty tophi in the intervertebral disc space. Acta neurochirurgica 144: 205-207, 2002

18. Yen PS, Lin JF, Chen SY, Lin SZ: Tophaceous gout of the lumbar spine mimicking infectious spondylodiscitis and epidural abscess: Mr imaging findings. J Clin Neurosci 12:44-46, 2005 